\title{
Prescriber Attitudes Toward Implementation of Pharmacogenomic Testing in a Family Medicine Residency Program
}

\author{
Alyssa Gallipani, PharmD, BCACP | Rebecca Cope, PharmD, BCACP | Briann Fischetti, PharmD, \\ BCACP | Sherly Abraham, MD | Arvind Ankireddypalli, MD
}

PRIMER. 2018;2:22.

Published: 10/11/2018 | DOI: 10.22454/PRiMER.2018.493524

\section{Abstract}

Introduction: Few qualitative studies have explored the attitude of prescribers towards the implementation of pharmacogenomic testing in the family medicine (FM) setting, and none among FM residents. The purpose of this study was to describe the level of engagement and interest in the implementation of pharmacogenomic education and testing in an FM clinic within a residency program.

Methods: A qualitative study utilizing semistructured interviews was conducted among prescribers within the FM clinic at The Brooklyn Hospital Center (TBHC). Voluntary prescribers included FM residents and attendings. No prescribers were excluded. Prior to the interview, informational sheets about pharmacogenomics were provided to standardize participant knowledge base. The research team created an interview guide of specific open-ended questions. Interviews were audio recorded and transcribed until a point of saturation was achieved. Transcripts of interviews served as data for analysis. Coding and analysis were performed to develop a hypothesis. No formal statistical analysis was required.

Results: Of the total 28 providers eligible for participation, 15 were recruited and interviewed (53\% response rate). Based on analysis of interview data, four key conceptual concerns emerged regarding benefits and risks of testing, feasibility, accessibility, and modification of FM residency training curricula.

Conclusion: Positive attitudes and perceptions provide support for pharmacogenomic education and testing to be incorporated into FM residency curricula. Addressing practical barriers, such as curricular education and training, will allow for expansion of such initiatives in the future.

\section{Introduction}

Pharmacogenomic testing aims to identify genetic risk for treatment nonresponse or adverse medication effects to determine optimal pharmaceutical therapy. ${ }^{1}$ The first identification of genetic influences on drug response dates back to the 1950s, when links between inheritance or ethnicity and aberrant drug responses were discovered. ${ }^{2}$ Pharmacogenomic testing contributes to optimal pharmacotherapy management for a variety of conditions, including cardiovascular disease, venous thromboembolism, and cancers. Its use for prescribing in family medicine (FM) practices potentially helps to avoid adverse effects and ineffective treatment. For example, testing for presence of the $H L A-B^{\star} 1502$ gene can prevent occurrence of Stevens-Johnson syndrome (SJS) and toxic epidermal necrolysis (TEN) in patients being treated with carbamazepine. ${ }^{3}$

There is a growing interest in and awareness of the importance of pharmacogenomics in medical practice. However, 
providers have expressed concerns regarding implementation of testing in routine care. ${ }^{3}$ Documented barriers include lack of training, reimbursement, and uncertain proof of improved outcomes. ${ }^{4}$ Although the field has grown tremendously, there has been minimal uptake of pharmacogenomic services in the primary care setting. Only $7 \%$ of hospitals in the United States offer routine pharmacogenomics testing services. ${ }^{4}$ Despite this, few studies have explored the attitude of FM prescribers towards implementation in FM settings, and none among FM residents. ${ }^{1,5,6}$ The purpose of this study was to describe the level of engagement and interest in the implementation of pharmacogenomic testing among health care providers in an FM residency program. Training and engagement of the next generation of residents is imperative to expansion and implementation of pharmacogenomic testing.

\section{Methods}

This qualitative study uses semistructured interviews of prescribers practicing in the FM Clinic at The Brooklyn Hospital Center (TBHC) in Brooklyn, New York. ${ }^{7}$ Interviews were conducted from January 2017 through February 2017. The institutional review board at TBHC approved this study.

Participants were recruited from the FM clinic at weekly clinician meetings. All prescribers in this setting were eligible, and none were excluded. Subjects who agreed to the research participated in a semistructured, open-ended interview. The interviews were conducted over a 2-month period by one member of the research team. All interviews were audio recorded and identified using subject code numbers. Prior to the interview, participants were provided a one-page document containing basic information on pharmacogenomic testing to standardize the knowledge base. Comprehension was not assessed. Topics covered included the purpose of pharmacogenomics testing and examples of clinical use.

The team created an interview guide (Table 1) that listed open-ended questions to be probed in a particular order. The interview guide was piloted for ease of comprehension. These data were not included in the final results.

Interviews were transcribed verbatim in Microsoft Word and verified with the research committee. Team members who performed the transcription did not participate in the coding process. Transcripts were made available to all participants upon request. An inductive, qualitative content analysis was performed simultaneously with interviewing to determine a point of saturation. Open coding was performed by the same two members of the team through simultaneous reading of each transcript to determine codes that emerged from the text. Codes were revised as data became available. The larger research team met to group similar codes into mutually exclusive categories. These categories were used to develop key conceptual concerns based on the emerging themes. Multiple team members participated in the coding process in attempt to mitigate subjective bias.

\section{Results}

Baseline demographic information and quotes representing the four key conceptual concerns identified are presented in Tables 2 and 3, respectively. Participants believed that pharmacogenomic testing offers advantages by providing precise information to optimize pharmacotherapy. This allows prescribers to transition from broad population-based treatments to patient-specific therapies. Despite this favorable view, participants expressed concerns of clinical validity, patient health literacy, cost-effectiveness, clinical utility, and safeguarding of information.

Implementation of pharmacogenomic services may lead to concerns of feasibility and time constraints in the workplace. However, a majority of interviewees felt that the additional time required to perform and interpret pharmacogenomics lab results would be worthwhile. Respondents also expressed beliefs that FM practitioners should serve as the gatekeeper of pharmacogenomic testing. Participants expressed a desire to collaborate with specialists and clinical pharmacists to make these medication-related modifications.

Participants felt that adjustments to FM residency curricula are necessary before pharmacogenomic services can be widely implemented in primary care settings. They expressed a desire for training on topics of clinical use and resources since educational exposure is minimal. Due to the structure of residency training, residents noted that 
opportunities to utilize pharmacogenomic services may depend on the attending physicians within the program. Therefore, it was suggested that education target all FM prescribers, including attending physicians.

\section{Discussion}

To our knowledge, this is the first study to address FM residents' attitudes and perceptions regarding pharmacogenomic testing implementation. Participants in our study had an overall favorable view of pharmacogenomics. However, due to minimal theoretical and clinical exposure during residency training, they were unable to make a clear analysis of the risks and benefits of such services. This corresponds to findings from similar studies among nontrainees that showed overlapping results regarding lack of knowledge, education, coursework and training. ${ }^{8,9}$ Prescribers also indicated similar concerns of insufficient time to counsel patients, especially those with low health literacy. ${ }^{10,11}$

One noticeable difference from previous results is that our participants did not expand on ethical and legal concerns that may exist with pharmacogenomic testing. The enthusiasm voiced by our participants may be due in part to lack of experience handling this type of sensitive information. We recommend for any educational curriculum to include a debate on the benefits and risks of pharmacogenomic testing, including ethical and legal implications, to facilitate critical thinking about these issues.

Based on our results, we recommend that such a curriculum include formal didactic lectures and/or webinars about DNA structure, concepts of drug absorption, distribution, metabolism and excretion, available resources, and application in a clinical setting. We recommend providing opportunities for practical experiences, interdisciplinary collaboration, counseling patients at various levels of health literacy, and ordering, interpreting, and discussing test results. All educational experiences should be designed for any level of provider (resident or attending physician). When possible, we recommend including clinical pharmacists in the design and delivery of these educational interventions.

This study has several limitations. Due to the qualitative design of this study, data cannot be generalized to a larger population or prescribers from other facilities. Although a majority of FM residents at this institution participated in the interviews, the population size is small and may also limit generalizability. Next, due to the open-natured study design, it is possible that all relevant data were not gathered. Lastly, responses collected were highly dependent on the clinical experience and perspectives of study participants. Since all study participants practice at the same facility, they may share similar experiences and understandings.

Pharmacogenomic services are projected to be more readily available in the next 5 to 10 years. Therefore, the current generation of residents would benefit from exposure to the clinical validity and utility of various pharmacogenomic tests during their years of training. There is a responsibility to the providers of the future to fill these educational and knowledge gaps.

\section{Conclusion}

Positive attitudes and perceptions provide support for pharmacogenomic education and testing to be incorporated into FM residency curricula. Addressing practical barriers such as curricular education and training will allow for expansion of such initiatives in the future.

\section{Tables and Figures}


Table 1: Interview Guide

\section{Introductory Knowledge Base}

1. How were you first introduced to pharmacogenomics? Where have you learned about pharmacogenomic testing? Where have you heard the term before?

2. How would you define the term "pharmacogenomics"?

3. If you received a pharmacogenomic test result for a patient, where would you go to learn how to interpret the result?

4. What medications would you consider using pharmacogenomic testing for?

\section{Applicability to Practice}

1. How would pharmacogenomic information in a patient's chart be helpful to you? How would you utilize this information if available?

2. Would you first review pharmacogenomic results before prescribing a new medication?

3. Which type of specialist or generalist do you think is best equipped to order and interpret pharmacogenomic results and why? Should use of pharmacogenomic testing be specialty based (ie, cardiologists test for cardiovascular medications, like clopidogrel)?

\section{Barriers}

1. What concerns do you have about pharmacogenomic testing? What barriers are you concerned about?

2. How confident are you in using, ordering, or counseling patients regarding pharmacogenomic testing? What would you need to be confident?

3. How confident are you in communicating with other healthcare providers about pharmacogenomic testing?

4. What suggestions do you have about how to integrate pharmacogenomic testing into clinical practice?

\section{Attitudes and Perceptions}

1. What are your thoughts about incorporating education on pharmacogenomic testing into a family medicine residency program?

2. Do you see room for implementation in the family medicine clinic?

Table 2: Baseline Demographics of Study Participants ( $\mathrm{N}=15)$

\begin{tabular}{|c|c|}
\hline Demographics & n (\%) \\
\hline Gender & $6(40)$ \\
Male & $9(60)$ \\
\hline Race & \\
African American & $1(7)$ \\
White (non-Hispanic) & $3(20)$ \\
Other & $6(40)$ \\
Asian, Asian American, Pacific Islander & $5(33)$ \\
\hline Previous practice in academic facility & $4(27)$ \\
\hline Current Title & \\
\hline First-year medical resident & $5(33)$ \\
Second-year medical resident & $6(40)$ \\
Third-year medical resident & $1(7)$ \\
Family medicine attending physician (MD) & $1(7)$ \\
\hline Family medicine attending physician (DO) & \\
\hline Year of Graduation & $4(27)$ \\
\hline 2000-2009 & $11(73)$ \\
\hline
\end{tabular}


Table 3: Key Conceptual Concerns

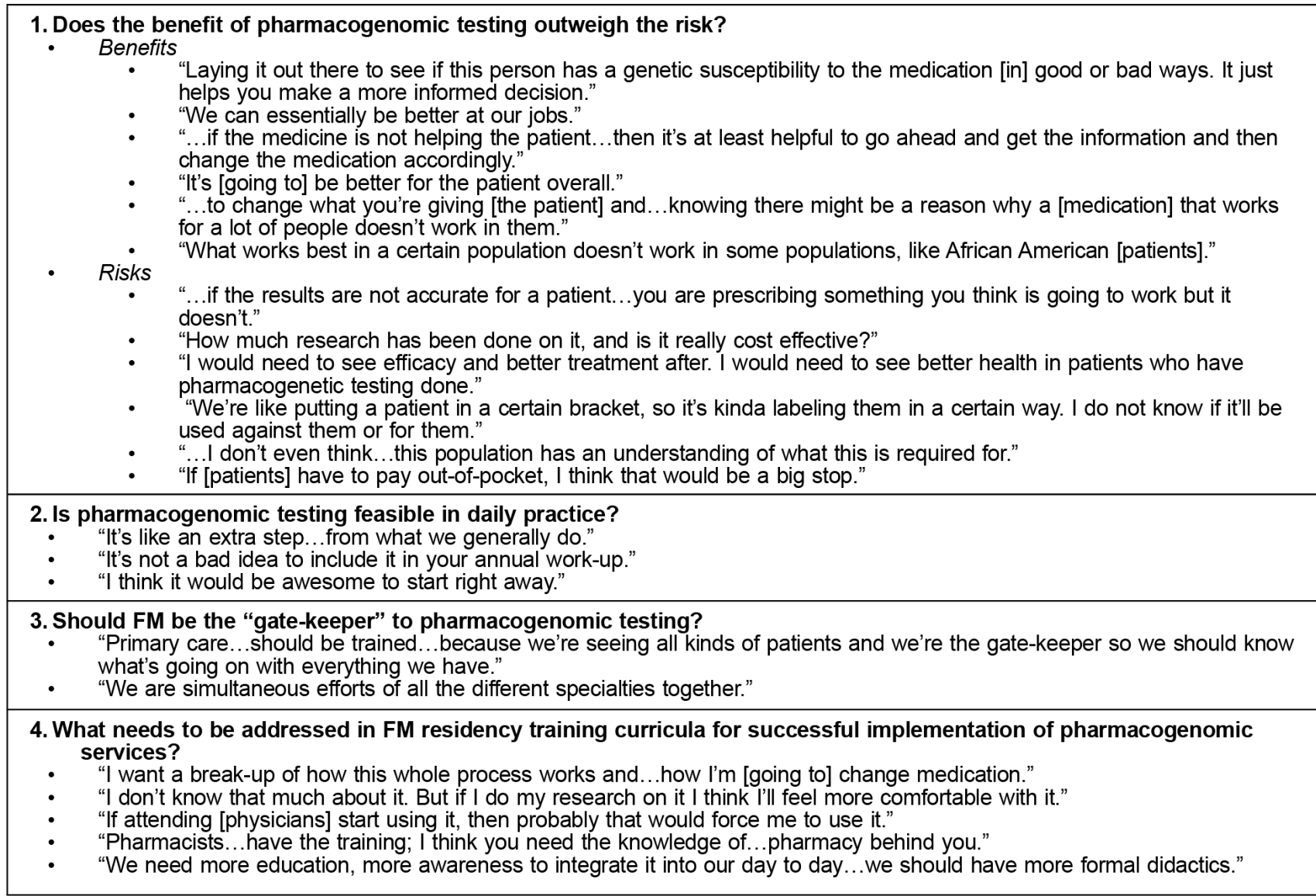

\section{Acknowledgments}

This study was presented in poster format at the 2017 Society of Teachers of Family Medicine Annual Spring Conference, May 5-9, San Diego, CA.

\section{Corresponding Author}

Alyssa Gallipani, PharmD, BCACP

Fairleigh Dickinson University School of Pharmacy and Health Sciences, 230 Park Avenue, Florham Park NJ 07932. 862-212-2486.

alyssa.j.gallipani@gmail.com

\section{Author Affiliations}

Alyssa Gallipani, PharmD, BCACP - Fairleigh Dickinson University School of Pharmacy and Health Sciences, Florham Park, NJ. RWJBarnabas Health-Corporate Pharmacy, Livingston, NJ.

Rebecca Cope, PharmD, BCACP - Long Island University, Arnold and Marie Schwartz College of Pharmacy and Health Sciences.

Briann Fischetti, PharmD, BCACP - Long Island University, Arnold and Marie Schwartz College of Pharmacy and Health Sciences

Sherly Abraham, MD - The Brooklyn Hospital Center, Family Medicine.

Arvind Ankireddypalli, MD - The Brooklyn Hospital Center, Family Medicine.

\section{References}

1. Haga SB, O'Daniel JM, Tindall GM, Mills R, Lipkus IM, Agans R. Survey of genetic counselors and clinical 
geneticists' use and attitudes toward pharmacogenetic testing. Clin Genet. 2012;82(2):115-120.

https://doi.org/10.1111/j.1399-0004.2012.01848.x

2. Evans WE, Relling MV. Moving towards individualized medicine with pharmacogenomics. Nature. 2004;429(6990):464-468. https://doi.org/10.1038/nature02626

3. Johnson SG. Leading clinical pharmacogenomics implementation: advancing pharmacy practice. Am J Health Syst Pharm. 2015;72(15):1324-1328. https://doi.org/10.2146/ajhp140613

4. Johnson JA, Weitzel KW. Advancing pharmacogenomics as a component of precision medicine: how, where, and who? Clin Pharmacol Ther. 2016;99(2):154-156. https://doi.org/10.1002/cpt.273

5. Zgheib NK, Arawi T, Mahfouz RA, Sabra R. Attitudes of health care professionals toward pharmacogenetic testing. Mol Diagn Ther. 2011;15(2):115-122. https://doi.org/10.1007/BF03256401

6. Bernhardt BA, Zayac C, Gordon ES, Wawak L, Pyeritz RE, Gollust SE. Incorporating direct-to-consumer genomic information into patient care: attitudes and experiences of primary care physicians. Per Med.

2012;9(7):683-692. https://doi.org/10.2217/pme.12.80

7. Silverman D, ed. Qualitative Research. 4th ed. Thousand Oaks, CA: SAGE Publications Inc; 2016.

8. Unertl KM, Field JR, Price L, Peterson JF. Clinician perspectives on using pharmacogenomics in clinical practice. Per Med. 2015;12(4):339-347. https://doi.org/10.2217/pme.15.10

9. Haga SB, Carrig MM, O'Daniel JM, et al. Genomic risk profiling: attitudes and use in personal and clinical care of primary care physicians who offer risk profiling. J Gen Intern Med. 2011;26(8):834-840. https://doi.org /10.1007/s11606-011-1651-7

10. Raghavan S, Vassy JL. Do physicians think genomic medicine will be useful for patient care? Per Med. 2014;11(4):424-433. https://doi.org/10.2217/pme.14.25

11. Haga SB, Tindall G, O'Daniel JM. Professional perspectives about pharmacogenetic testing and managing ancillary findings. Genet Test Mol Biomarkers. 2012;16(1):21-24. https://doi.org/10.1089/gtmb.2011.0045

Copyright $\odot 2018$ by the Society of Teachers of Family Medicine 